\title{
On optimizing the build orientation problem using genetic algorithm
}

Cite as: AIP Conference Proceedings 2116, 220006 (2019); https://doi.org/10.1063/1.5114224

Published Online: 24 July 2019

Marina A. Matos, Ana Maria A. C. Rocha, and Ana I. Pereira

\section{Conference Proceedings}

Get $30 \%$ off all print proceedings!

\section{Enter Promotion Code PDF-30 at checkout}




\title{
On Optimizing the Build Orientation Problem using Genetic Algorithm
}

\author{
Marina A. Matos ${ }^{1, b)}$, Ana Maria A.C. Rocha ${ }^{1, c)}$ and Ana I. Pereira ${ }^{1,2, a)}$ \\ ${ }^{1}$ Algoritmi Research Centre, University of Minho, 4710-057 Braga, Portugal. \\ ${ }^{2}$ Research Centre in Digitalization and Intelligent Robotics (CeDRI), Polytechnic Institute of Bragança, Portugal.

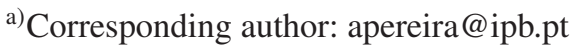 \\ b) aniram@live.com.pt \\ c)arocha@dps.uminho.pt
}

\begin{abstract}
Build orientation is a critical issue in Additive manufacturing (AM), where three-dimensional objects are created layerby-layer directly from a 3D CAD model, since it is associated with the object accuracy, the number of supports required and the processing time to produce the object. Finding the best build orientation in the AM will reduce, significantly, the building costs and will improve the object accuracy. This work presents the solutions obtained by the Genetic Algorithm (GA) in solving the part build orientation optimization problem, considering the staircase effect, support area characteristics and the building time of four models. Preliminary experiments show that GA gives competitive results in solving the build orientation problem when compared with other metaheuristics.
\end{abstract}

\section{INTRODUCTION}

Additive manufacturing (AM) is a technology that builds 3D objects by adding ultrathin layers of material, one by one, for constructing desired products. Currently, AM is being used to make end-use products for engineers and designers in the aircraft, dental restorations, medical implants, automobiles, and even fashion areas [1]. One of the current challenges is the reduction of construction time through adoption of rapid prototyping technologies, whose performance can depend on the way parts are oriented on the build platform. Therefore, each part should be appropriately oriented to achieve better surface quality and either minimal support structure or lower building time in order to result in a process more environmental friendly since it will require less energy consumption and material waste [2].

A number of studies have been carried out in the problem of selecting a building direction given a 3D CAD model. A proposal based on the determination of the optimal part orientation when minimizing the build cost can be seen in [3]. Authors used various criteria like stair step error, build height, volume of supports, stability of object, among others, to determine the optimal part build orientation for any rapid prototyping process. A part orientation optimization model considering the building time, material usage, surface finish, interior geometry, strength characteristics and related parameters by the genetic algorithm is presented in $[4,5]$. The literature suggests criteria to be considered for optimal build orientation such as the build height, staircase effect, volume of support structures and part area in contact with support structures $[5,6,7,8]$.

In this study, we aim to analyze the performance of the Genetic Algorithm (GA) to solve the build orientation optimization problem in additive manufacturing, using four models.

\section{PROBLEM FORMULATION}

The finish surface of an object obtained through additive manufacturing process is highly important. Different measures to determine the best build orientation for an improvement of the surface finish can be considered, such as the total volume of support material used, the total area of contact of the object with the external support structure, the quality of selected faces that are subjected to the staircase effect and are in contact with the supports or the height of

International Conference of Numerical Analysis and Applied Mathematics (ICNAAM 2018)

AIP Conf. Proc. 2116, 220006-1-220006-5; https://doi.org/10.1063/1.5114224

Published by AIP Publishing. 978-0-7354-1854-7/\$30.00 
the part in the build direction. These orientation characteristics can be individually used to rank a selection of possible orientations according to minimum build height, minimum volume of support structures, minimum area in contact with extra support structures or maximum accuracy of total surface area (staircase effect) or selected facets [5, 7]. The best selection of the build orientation model will improve the surface accuracy of the object, minimize the supports needed and the production time, and, consequently, the build costs [3].

The volumetric error, VE, to be minimized in each layer stem from staircase effect is given by

$$
V E=\sum_{j} \frac{t^{2}\left|d^{T} n_{j}\right| A_{j}}{2},
$$

where $t$ is the slicing height, $n_{j}$ is the unit normal vector of the $j$ triangle facet, $A_{j}$ is its area and $d$ is the unit normal vector of the building direction [9].

Another measure related to the optimal build orientation is the quantity of supports used, measured by the support area or support volume. The computation of the support volume is very complex depending on the convexity or not of the object whereas the support area is easier to be computed and is related to the total area of the downward-facing facets. Zhao in [9] reported that the support area have more significant impact on the object accuracy than the support volume. The support area, SA, to be minimized is expressed as follows

$$
S A=\sum_{j} A_{j}\left|d^{T} n_{j}\right| \delta
$$

where $\delta=1$ if $d^{T} n_{j}<0$ and $\delta=0$ if $d^{T} n_{j}>0$ is a threshold function.

The other measure that could be used to determine the optimal part build orientation is the building time without compromising the surface quality. Canellidis et al. [2] considered that the building time includes the time required to manufacture the object as well as the time required for support removal and surface finishing. However, Zhao [9] considered the building time as the preparation time that includes the time required to move down the platform during the re-coating, the scraping time and other preparation times. Since the preparation time is dependent on the total number of layers, and at the same time the number of layers is dependent on the height of part building direction, then the minimization of the height of the part building direction and, consequently, the total number of layers, can reduce the part building time. Hence, the building time, BT, to be minimized can be expressed as follows

$$
B T=\max \left(d^{T} v_{1}, d^{T} v_{2}, \ldots, d^{T} v_{n}\right)-\min \left(d^{T} v_{1}, d^{T} v_{2}, \ldots, d^{T} v_{n}\right)
$$

where $v_{i}$ are the vertex triangle facets.

The formulation of the build orientation optimization problem is given by the rotation along the $x$ and $y$ axis

$$
\begin{array}{ll}
\min & f(x, y) \\
\text { s.t. } & 0 \leq x, y \leq 180
\end{array}
$$

where $f(x, y)$ is given by (1) for the volumetric error, (2) for the support area and (3) for the part building time.

\section{EXPERIMENTAL RESULTS}

The numerical experiments involve the optimization of the build orientation problem using three different measures: the volumetric error, the support area and the part building time. The models used in the experiments are the Rear panel, Air duct, Rocket shot and 45 degree. Before optimization, the objects considered a slicing along the Z-axis with $5 \mathrm{~mm}$ height, except for the Air duct that used a slicing with $2 \mathrm{~mm}$ height. In this section, we report the numerical results obtained by Genetic Algorithm from the Matlab Global Optimization Toolbox ${ }^{\mathrm{TM}}$ (release 2017b).

For a fair comparison with the results obtained in [10] the population size used in the GA algorithm was set to 10 and in the stopping criterion the maximum number of function evaluations allowed was 500. In the experiments, 10 runs were performed for each model. Table 1 presents the different solutions, $(x, y)$ in degrees, obtained by GA for each model considered for each objective function. The obtained solutions are consistent with the main idea of the measures definition of VE, SA and BT.

As can be concluded from Table 1 the GA obtained similar global solution values when compared with the solutions obtained by Electromagnetism-Like (EM) and Stretched Simulated Annealing (SSA) algorithms in [10], 
TABLE 1. GA results.

\begin{tabular}{|c|c|c|c|c|c|c|}
\hline \multirow[b]{2}{*}{ Problem } & \multicolumn{2}{|c|}{ VE } & \multicolumn{2}{|c|}{ SA } & \multicolumn{2}{|c|}{ BT } \\
\hline & $\left(x^{*}, y^{*}\right)$ & $f\left(x^{*}, y^{*}\right)$ & $\left(x^{*}, y^{*}\right)$ & $f\left(x^{*}, y^{*}\right)$ & $\left(x^{*}, y^{*}\right)$ & $f\left(x^{*}, y^{*}\right)$ \\
\hline \multirow[t]{2}{*}{ Rear panel } & $(90,0$ & $2.4 \times 10^{4}$ & $(90,0)$ & $8.6 \times 10^{2}$ & $(0,135)$ & $4.4 \times 10^{1}$ \\
\hline & $(100,170)$ & $5.9 \times 10^{3}$ & $(90,180)$ & $8.6 \times 10^{2}$ & $(180,45)$ & $4.4 \times 10^{1}$ \\
\hline \multirow[t]{2}{*}{ Air duct } & $(0,0)$ & $1.8 \times 10^{4}$ & $(0,0)$ & $4.3 \times 10^{3}$ & $(100,90)$ & $5.2 \times 10^{1}$ \\
\hline & - & 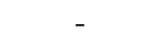 & - & - & $(75,95)$ & $5.7 \times 10^{1}$ \\
\hline \multirow[t]{3}{*}{ Rocket shot } & $(0,0)$ & $2.7 \times 10^{4}$ & $(0,0)$ & $9.2 \times 10^{2}$ & $(90,45)$ & $4.5 \times 10^{1}$ \\
\hline & - & - & - & - & $(90,135)$ & $4.5 \times 10^{1}$ \\
\hline & - & 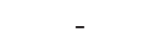 & - & - & $(45,90)$ & $6.1 \times 10^{1}$ \\
\hline \multirow[t]{2}{*}{45 degree } & $(90,135)$ & $4.8 \times 10^{5}$ & $(170$ & $2.4 \times$ & $(0,0)$ & $1.3 \times 10^{2}$ \\
\hline & 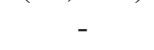 & - & $(90,135)$ & $1.9 \times 1$ & - & 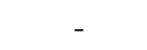 \\
\hline
\end{tabular}

although the GA found fewer global solutions. Besides that GA failed to have a success rate as good as EM and SSA. For example, in the Rear panel model only 2 among the 10 runs were able to reach the global optimal $\left(90^{\circ}, 0^{\circ}\right)$ and $\left(90^{\circ}, 180^{\circ}\right)$ for VE measure. In addition, for SA measure, the GA converged to the two global optimal $\left(90^{\circ}, 0^{\circ}\right)$ and $\left(90^{\circ}, 180^{\circ}\right)$ angles. In fact the values in the Table 1 were rounded to integer values.

To evaluate the impact of the different measures identifying the $(x, y)$ on the 3D printer, Figure 1 presents the direction provided by the GA when optimizing the build orientation problem of the models. We can see that GA found different orientations for the different objective functions. From Figure 1 for the 45 degree model for SA measure GA found a local solution at $\left(170^{\circ}, 90^{\circ}\right)$. In the case of the Rocket shot for the objective function BT the algorithm also found a local solution $\left(45^{\circ}, 90^{\circ}\right)$, since this solution places the piece in an orientation that has a greater height.

\section{CONCLUSIONS}

In this paper, an experimental study to solve the build orientation optimization problem is presented. Numerical experiments have been carried out using the GA when determining the best orientation to improve the surface accuracy of four 3D CAD models using three different measures, the volumetric error, the support area and the building time. Although GA has effectively solved the orientation optimization problem, it has identified fewer global solutions in each model/measure than other global stochastic methods in the literature. For future work we intend to solve the build orientation problem as a multiobjective problem.

\section{ACKNOWLEDGMENTS}

This work has been supported and developed under the FIBR3D project - Hybrid processes based on additive manufacturing of composites with long or short fibers reinforced thermoplastic matrix (POCI-01-0145-FEDER-016414), supported by the Lisbon Regional Operational Programme 2020, under the PORTUGAL 2020 Partnership Agreement, through the European Regional Development Fund (ERDF). This work was also supported by COMPETE: POCI-01-0145-FEDER-007043 and FCT - Funda£o para a Ciłncia e Tecnologia within the Project Scope: UID/CEC/00319/2013. 

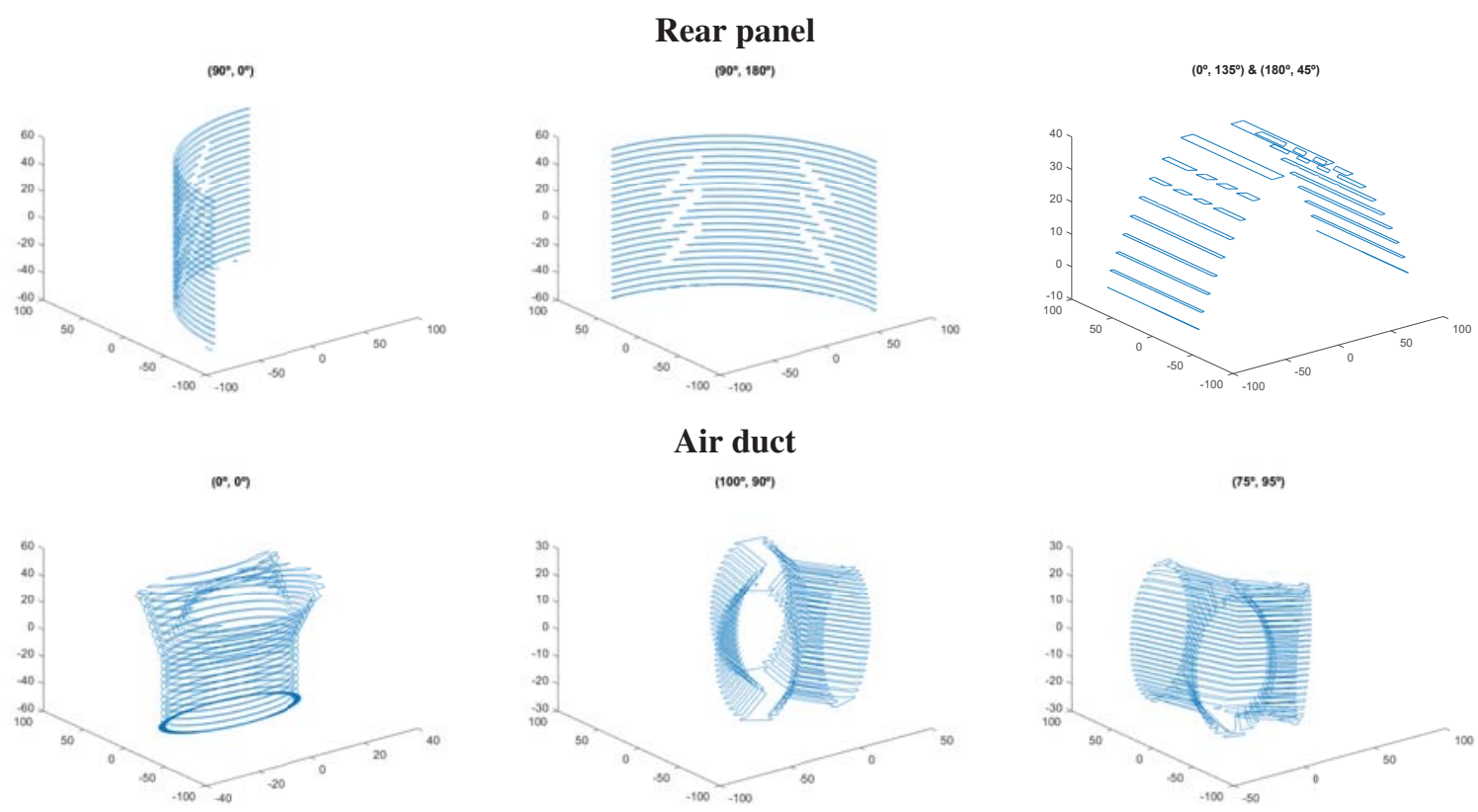

Air duct

$\left({ }^{100 \%}, 90^{\circ}\right)$
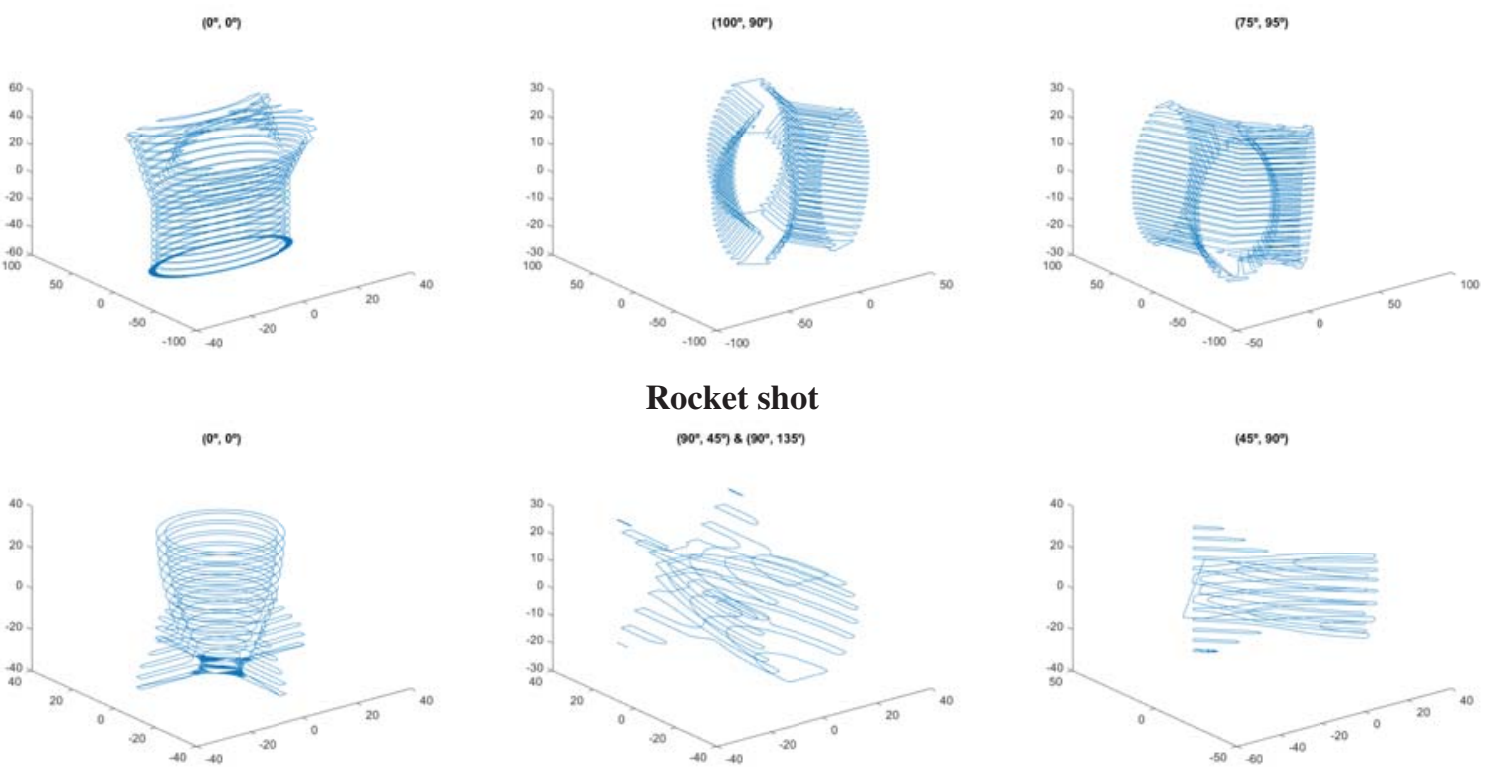

Rocket shot
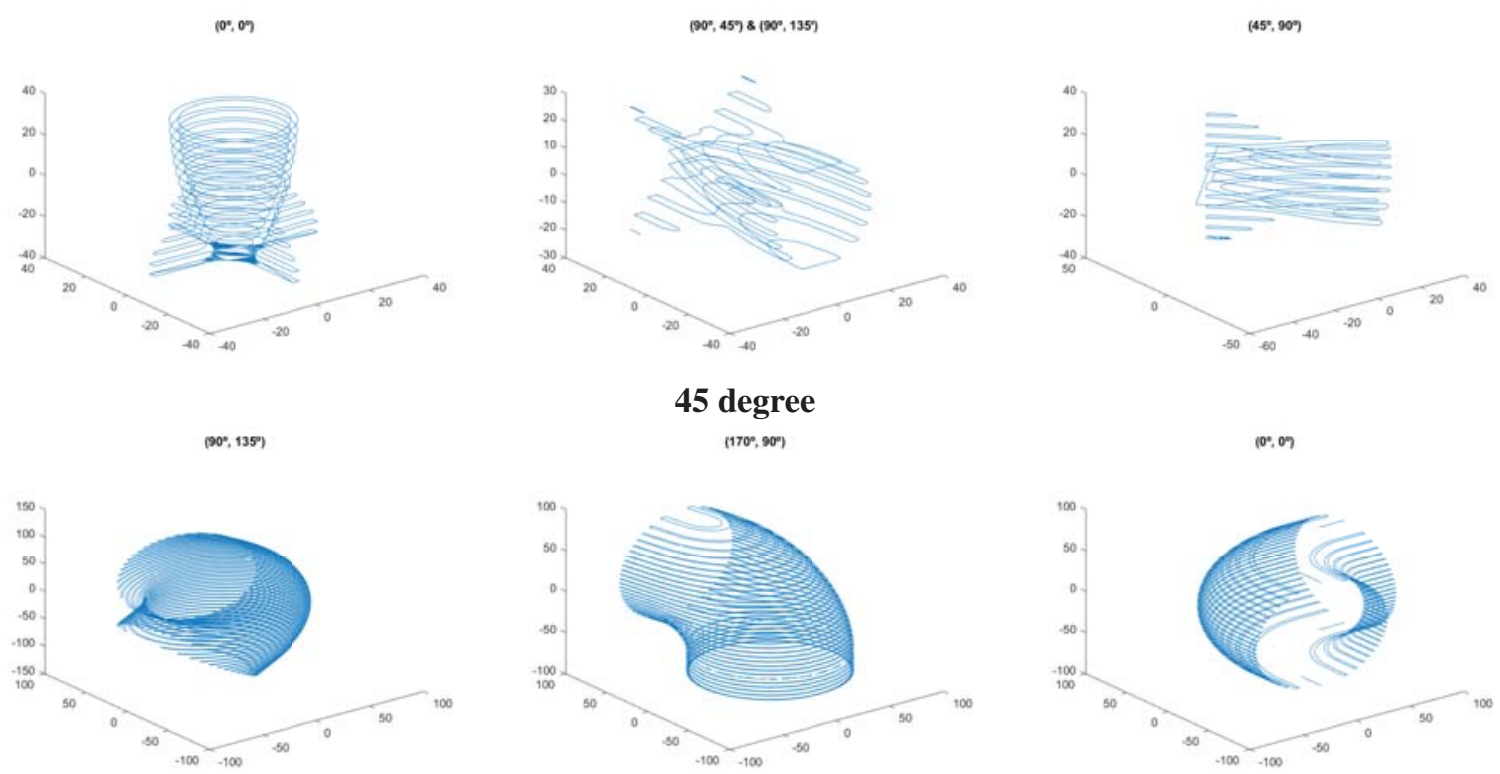

FIGURE 1. Plots of GA solutions. 


\section{REFERENCES}

[1] I. Khana, A. Mateus, K. Lorgerc, G.R. Mitchella, Procedia Manufacturing 12, 89-95 (2017).

[2] V. Canellidis, V. Dedoussis, N. Mantzouratos, S. Sofianopoulou, Computers in Industry 57, 424-36 (2006).

[3] P. Alexander, S. Allen, D. Dutta, Computer-Aided Design, 30, 343-356 (1998).

[4] A.M. Phatak, S.S. Pande, Journal of Manufacturing Systems, 31, 395-402 (2012).

[5] L. Villalpando, H. Eiliat, , R.J. Urbanic, Procedia CIRP 17, 800-805 (2014).

[6] V. Canellidis, J. Giannatsis, V. Dedoussis, International Journal of Advanced Manufacturing Technology 45, 714-730 (2009).

[7] A.S. Gogate and S.S.Pande, International Journal of Production Research 46, 5607-5631 (2008).

[8] S.H. Masood, W. Rattanawong, P. Iovenitti, Journal of materials processing technology, 139, 110-116 (2003).

[9] J. Zhao, "Determination of optimal build orientation based on satisfactory degree theory for RPT" in Ninth International Conference on Computer Aided Design and Computer Graphics (CAD-CG'05), 6 pages, Hong Kong, China (2005).

[10] A.M.A.C. Rocha, A.I. Pereira and A.I. Vaz, "Build Orientation Optimization Problem in Additive Manufacturing" in Computational Science and Its Applications - ICCSA 2018, Lecture Notes in Computer Science 10961, edited by O. Gervasi et al. (Springer International Publishing 2018), pp. 669-682. 